Canadian Journal of Regional Science

Revue canadienne des sciences régionales

\title{
Les stratégies organisationnelles déployées par les aînés en vue d'atténuer les effets de l'érosion des services de proximité en milieu rural dévitalisé et leur contribution au renforcement des capacités
}

\section{Majella Simard}

Volume 44, numéro 3, 2021

Innovations et territoires face aux inégalités

Soumis : 26 novembre 2019

Accepté : 9 novembre 2020

URI : https://id.erudit.org/iderudit/1086214ar

DOI : https://doi.org/10.7202/1086214ar

Aller au sommaire du numéro

Éditeur(s)

Canadian Regional Science Association / Association canadienne des sciences régionales

ISSN

0705-4580 (imprimé)

1925-2218 (numérique)

Découvrir la revue

Citer cet article

Simard, M. (2021). Les stratégies organisationnelles déployées par les aînés en vue d'atténuer les effets de l'érosion des services de proximité en milieu rural dévitalisé et leur contribution au renforcement des capacités. Canadian Journal of Regional Science / Revue canadienne des sciences régionales, 44(3), 139-149. https://doi.org/10.7202/1086214ar

\section{Résumé de l'article}

Les inégalités constituent depuis longtemps un sujet prisé par les spécialistes des sciences sociales. En milieu rural, les mutations survenues depuis la deuxième moitié $\mathrm{du} \mathrm{XX}^{\mathrm{e}}$ siècle ont eu pour effet d'accentuer les inégalités au sein des milieux de petite taille démographique éloignés des villes, dont de vastes segments sont aux prises avec une insuffisance de services. L'objectif de cet article consiste à identifier la nature des stratégies organisationnelles mises en place par les différents intervenants et les aînés à la suite de la perte des services de proximité et leurs impacts sur la qualité de vie des personnes âgées en milieu rural fragile. Notre analyse s’appuie sur le modèle de gérontologie environnementale mis en exergue avec celui de renforcement des capacités d'apprentissage et le concept d'équité territoriale. L'étude de cas multiples est la méthode retenue aux fins de notre analyse, laquelle est couplée à l'approche des représentations sociales. 


\title{
CANADIAN JOURNAL OF REGIONAL SCIENCE

\author{
REVUE CANADIENNE DES \\ SCIENCES REGIONALES
}

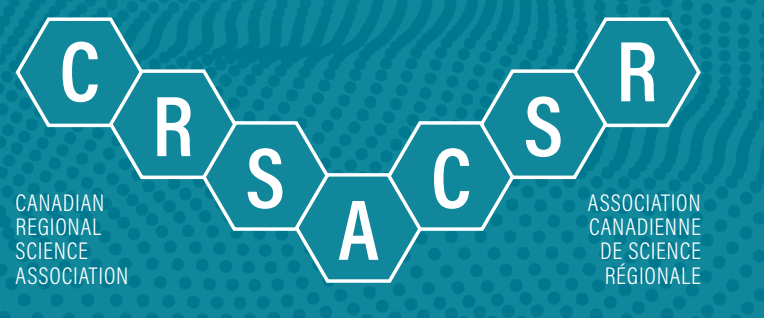

\section{LES STRATÉGIES ORGANISATIONNELLES DÉPLOYÉES PAR LES AÎNÉS EN VUE D'ATTÉNUER LES EFFETS DE L'ÉROSION DES SERVICES DE PROXIMITÉ EN MILIEU RURAL DÉVITALISÉ ET LEUR CONTRIBUTION AU RENFORCEMENT DES CAPACITÉS}

\section{Majella Simard}

\author{
Majella Simard \\ Professeur \\ Département d'histoire et de géographie \\ Édifice des Arts et des sciences sociales \\ Université de Moncton \\ Moncton (Nouveau-Brunswick) \\ E1A 3E9 \\ majella.simard@umoncton.ca
}

Soumis : 26 novembre 2019

Accepté: 9 novembre 2020

Résumé: Les inégalités constituent depuis longtemps un sujet prisé par les spécialistes des sciences sociales. En milieu rural, les mutations survenues depuis la deuxième moitié du XXe siècle ont eu pour effet d'accentuer les inégalités au sein des milieux de petite taille démographique éloignés des villes, dont de vastes segments sont aux prises avec une insuffisance de services. L'objectif de cet article consiste à identifier la nature des stratégies organisationnelles mises en place par les différents intervenants et les aînés à la suite de la perte des services de proximité et leurs impacts sur la qualité de vie des personnes âgées en milieu rural fragile. Notre analyse s'appuie sur le modèle de gérontologie environnementale mis en exergue avec celui de renforcement des capacités d'apprentissage et le concept d'équité territoriale. L'étude de cas multiples est la méthode retenue aux fins de notre analyse, laquelle est couplée à l'approche des représentations sociales.

Mots clés: innovation sociale, services, vieillissement, milieu rural, Nouveau-Brunswick 
Les inégalités constituent depuis longtemps un sujet prisé par les économistes, les sociologues et les géographes (Piketty, 2019; Badie \& Vidal, 2017; Atkinson, 2016). Elles affectent de différentes manières les individus et les diverses portions de l'espace géographique (Aubry \& Léger, 2015; Meloche, 2012; Dugas, 1994). Bien que depuis le XIXe siècle, les inégalités aient eu tendance à s'atténuer à l'échelle mondiale en raison d'un meilleur accès à l'éducation et aux progrès observés dans les domaines de la médecine, des transports et des communications, les mutations survenues depuis la deuxième moitié du XXe siècle ont eu pour effet d'accentuer les disparités territoriales aux échelons géographiques plus fins, particulièrement au sein des petites localités rurales éloignées des villes, dont de vastes segments sont aux prises avec une insuffisance de services, la faiblesse des niveaux de revenus, un chômage chronique et le vieillissement accéléré de leur population (Argoud, 2017; Dumont, 2012; Simard, 2003; Dugas, 1996). Au Nouveau-Brunswick, une province du Canada à dominante rurale, plusieurs localités de petite taille démographique ont été affectées par ces transformations structurelles. La forte dispersion de la population entraîne notamment une répartition inégale des services de proximité dans l'espace, lesquels sont aussi assujettis à une profonde restructuration (Simard et al., 2015). Ainsi, au cours des dernières années, nous avons assisté, au sein de plusieurs localités rurales du Nouveau-Brunswick, à la fermeture de coopératives alimentaires, de points de services des caisses populaires, d'écoles et de presbytères. Or, l'érosion de l'infrastructure de services apporte son lot de conséquences, tant positives que négatives, pour les populations plus vulnérables de la société et, en particulier, pour les personnes âgées résidant, au surplus, en milieu minoritaire francophone (Simard, 2019; 2015).

Comment les aînés qui résident en milieu rural fragile défavorisé et, de surcroît, en situation linguistique minoritaire, conjuguent-ils avec la dissolution progressive des services de proximité au sein de leur communauté? Quels sont les impacts de la perte de services en milieu rural défavorisé sur la qualité de vie des aînés, le renforcement des capacités et le tissu sociocommunautaire? Les stratégies organisationnelles mises en place par les intervenants sociaux et les aînés dans le but d'atténuer les effets liés à l'érosion de l'infrastructure de services peuvent-elles être considérées comme des innovations sociales? Telles sont les trois principales questions auxquelles nous tenterons de répondre dans le cadre de cet article, dont l'objectif consiste à identifier, au sein de deux localités rurales du Nouveau-Brunswick, la nature des stratégies organisationnelles mises en place par les intervenants sociaux et les personnes âgées à la suite de la perte progressive des services de proximité ainsi que les impacts de ces stratégies sur la qualité de vie des aînés.

Notre article se décline en quatre parties. Dans la première, nous esquissons notre cadrage théorique. La deuxième est consacrée aux aspects méthodologiques. La troisième présente les résultats de notre analyse. Pour chacune des deux localités à l'étude, nous dépeignons brièvement leur situation socioéconomique. Puis, nous identifions les manifestations et les conséquences liées à l'érosion des services de proximité. Nous terminons en identifiant les stratégies organisationnelles déployées par les intervenants sociaux et les aînés suite à la restructuration des services. Enfin, l'article se conclut par une discussion. Celle-ci reviendra sur les résultats de notre démonstration que nous confronterons à notre cadre conceptuel.
Notre analyse prend appui sur le modèle de gérontologie environnementale (Scheidt \& Schwartz, 2013; Wahl \& Weisman, 2003; Lawton, 1998; 1986) que nous mettrons en exergue avec celui de renforcement des capacités d'apprentissage (Jean, 2012; Davis, 2008) et le concept d'équité territoriale (Pech, Léon \& Dupraz, 2001). Mise de l'avant par le psychologue Powell Lawton (1923-2001), la gérontologie environnementale examine le phénomène du vieillissement dans une perspective multidisciplinaire favorisant la compréhension du rôle de l'environnement dans la détermination de la qualité de vie des aînés (Wiles et al., 2012; Iwarsson, 2005; Kending, 2003).

Quant au renforcement des capacités, il s'agit d'un paradigme qui s'inscrit dans la foulée des modèles de développement endogène ayant vu le jour dans les années 1980 (Jean, 2015; Simard, Diallo \& Tremblay, 2014). Le Programme des Nations Unies pour le développement (PNUD) le définit comme un «processus par lequel particuliers, organisations, et sociétés acquièrent, accroissent et entretiennent les aptitudes requises pour se fixer leurs propres objectifs de développement et pour les atteindre» (Davis, 2008: 3). Le renforcement des capacités s'incarne à travers différentes composantes qui, à l'instar de la gérontologie environnementale, favorisent l'amélioration de la qualité de vie des individus par la prise en charge de leur propre destinée et de celle de leur communauté (Besançon \& Chochoy, 2019; Tremblay, Klein \& Fontan, 2016). Parmi ces composantes, mentionnons: la participation citoyenne, la mobilisation sociale, le leadership, la résilience, le capital socioterritorial, la gouvernance, la dynamique relationnelle et communautaire, la concertation, le sentiment identitaire, l'empowerment et l'innovation sociale (Simard, 2018). À défaut de pouvoir toutes les analyser, nous retiendrons, dans le cadre de cet article, trois composantes, à savoir: la résilience, l'empowerment et l'innovation sociale, les deux premières' ayant une forte incidence sur la dernière.

La résilience est souvent présentée comme la «capacité de rebondir » face à l'adversité. Cette qualité fait appel à la fois à la fragilité et à la souplesse. Cyrulnik (1998), appuyé par Guedeney (1998), aborde la résilience comme un processus dynamique et positif, lequel est étroitement lié à la notion de développement (Simard, 2016). La définition de Tomkiewicz nous apparaît particulièrement éclairante. Ce dernier définit la résilience comme étant «la capacité d'une personne ou d'un groupe à se développer bien, à continuer à se projeter dans l'avenir, en présence d'événements déstabilisants, de conditions de vie difficiles, de traumatismes parfois sévères » (citée par Manciaux, 2004: 36).

Sur le plan étymologique, l'empowerment signifie «renforcer» ou «acquérir du pouvoir » (Von Bardeleben, 2016). Dès lors, l'empowerment fait référence à la capacité des individus (ou des communautés) à influencer, dans le cadre d'une situation donnée, le comportement des autres personnes dans l'optique d'atteindre un objectif commun, celui-ci correspondant le plus souvent à une volonté de prise en main des différents leviers du développement (El-Batal \& Joyal, 2015; Ninacs, 2012; 2002). L'empowerment conduirait donc les individus à se positionner en tant qu'acteurs du processus de transformation sociale de leur environnement suivant une perspective de justice sociale (Alberio, 2015; Ninacs, 2012; Grenier \& Simard, 2011). II prendrait la forme d'une action individuelle ou collective s'inscrivant au sein du territoire dans un contexte de transformation sociale, d'où son point de convergence avec le concept d'innovation sociale (Besançon et al., 2013).

Le Centre de recherche sur les innovations sociales (CRISES) définit cette dernière comme étant une «intervention initiée par des acteurs sociaux (un individu ou un groupe d'individus) pour répondre 
à un besoin (social, culturel, territorial) ou une aspiration, apporter une solution, profiter d'une opportunité d'action afin de modifier des relations sociales, de transformer un cadre d'action ou de proposer de nouvelles orientations afin d'améliorer la qualité et les conditions de vie d'une collectivité » (Bouchard, 2011: 7). Dès lors, l'innovation sociale est susceptible de produire une transformation sociale, c'està-dire «l'adoption de nouveaux arrangements sociaux, organisationnels ou institutionnels » (Klein, 2017: 13) servant à enclencher le processus de développement (Klein et al., 2016; Klein et al., 2009). Parce qu'il n'y a pas d'innovation sociale sans qu'il y ait une collaboration entre les acteurs provenant de milieux diversifiés permettant d'obtenir une meilleure compréhension des enjeux et des défis qui touchent les aînés, de développer des solutions et d'investir les expertises et les ressources nécessaires à la réussite du changement, l'innovation sociale nous apparaît tout indiquée afin de servir de concept fédérateur dans le cadre de cette contribution (Simard, 2018; Garon et al., 2014).

La question des services de proximité fait également intervenir, au sein des milieux ruraux, le concept d'équité qui a servi de fondement à l'analyse spatiale (Thiesse, 1994). II réfère à la dimension spatiale de la justice sociale (Bret, 2009) et, de manière plus spécifique, au principe selon lequel tout individu, quelle que soit sa localisation géographique, soit en mesure de bénéficier d'un accès équitable aux ressources physiques, humaines et matérielles disponibles sur un territoire donné (Lesourd, 2003; Pech, Léon \& Dupraz, 2001) et ce, dans la perspective de favoriser son épanouissement et de promouvoir un développement équilibré. Dans le domaine de la gérontologie environnementale, la notion d'équité «interroge les orientations des politiques publiques et leur capacité à prendre en compte et à soutenir des parcours de vieillissement à distance des modèles dominants »(Gucher, 2013: 19). La recherche de cette équité n'est pas la responsabilité exclusive des acteurs publics. Elle se déploie à travers le dynamisme sociocommunautaire et l'intensité du capital social qui caractérisent un territoire donné, d'où son rapport avec le renforcement des capacités (Chapon, 2013).

La fragilité réfère à l'existence de problèmes socioéconomiques, culturels, politiques et géographiques qui caractérisent certains milieux ruraux, notamment ceux de petite taille démographique et éloignés des villes. Hérités à la fois du passé et des dynamiques actuelles, ces problèmes se matérialisent, entre autres choses, par l'insuffisance de services, la décroissance démographique, le sous-emploi, la faiblesse des niveaux de revenus et l'émigration de la population, en particulier des jeunes et des personnes âgées, mais aussi des actifs (Simard, 2016; 2003). II importe de rappeler que «fragilité » et «dévitalité » désignent deux réalités fort différentes. Par exemple, une localité peut présenter certains signes de fragilité économique tout en étant en stabilité, voire en relative croissance sur le plan démographique. Quant à la fragilisation, elle renvoie au processus par lequel un milieu tend à se dégrader en raison de facteurs économiques, géographiques, historiques et sociaux tels que l'éloignement des pôles de développement, la dispersion de la population, la pauvreté des sols et du potentiel biophysique en général, l'épuisement ou la mauvaise gestion des ressources naturelles, la décroissance démographique, la perte de services et d'emplois, les fluctuations économiques, etc. Elle comporte ipso facto une dimension temporelle. Le processus engage l'avenir puisqu'il implique la perte et l'amenuisement de facteurs de développement tant physiques, humains, tangibles qu'intangibles (Simard, 2016).

Enfin, nous ferons référence, dans le cadre de cet article, à quatre phénomènes démographiques différents à la fois dans leurs causes et leurs conséquences (Dumont, 2018). Il s'agit de la gérontocrois- sance, définie par l'augmentation du nombre de personnes âgées au cours d'une période donnée, du vieillissement par le haut, c'està-dire l'augmentation de la proportion de personnes âgées parmi la population totale, de la juvénodécroissance, résultat de la diminution du nombre de jeunes (0 à 24 ans), et du vieillissement par le bas qui correspond à la diminution de la proportion de jeunes parmi la population totale.

\section{CADRAGE OPÉRATOIRE}

\section{Approche préconisée}

L'étude de cas multiples couplée à l'approche des représentations sociales est la méthode retenue aux fins de notre analyse. Deux localités rurales néo-brunswickoises, l'une en voie de fragilisation et en situation périurbaine et l'autre en situation de fragilité, nous ont servi de laboratoire d'investigation. II s'agit de Robertville et de Saint-Wilfred, deux districts de services locaux (DSL) ${ }^{2}$. Le choix de ces deux localités s'est effectué en concertation avec l'Association francophone des aînés du Nouveau-Brunswick, un organisme particulièrement impliqué pour tout ce qui concerne les droits, l'épanouissement et le bien-être des aînés francophones de la province. Ces milieux présentent des profils socioéconomiques différents. Toutefois, ils ont plusieurs dénominateurs communs. Situés dans la péninsule acadienne, ils comportent une population majoritairement francophone dans une province où cette dernière est minoritaire ${ }^{3}$. Sujets au vieillissement, ils font preuve, chacun à leur manière, d'un fort dynamisme sur le plan sociocommunautaire. Ils ont été lourdement affectés par la dissolution progressive de leurs services de proximité au cours des dernières années.

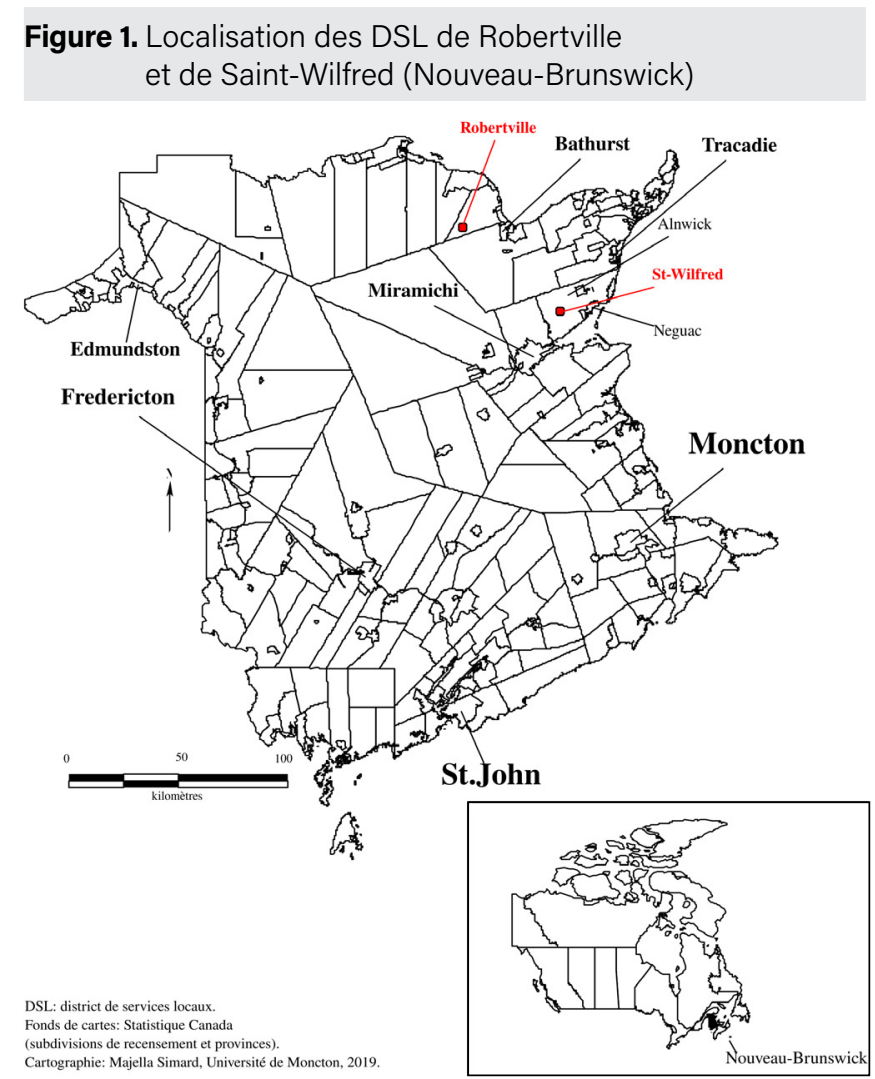

Cartographie Majella Simard Université de Moncton, 2019.

*Source : M. Simard

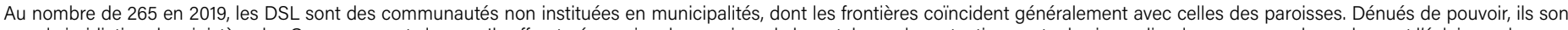

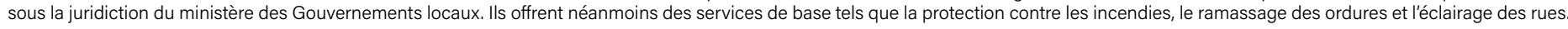
3 Cette proportion est de $92,4 \%$ dans le cas de Robertville et de 95,4\% dans celui de Saint-Wilfred par rapport à 31,6\% pour l'ensemble du Nouveau-Brunswick. 
Quant aux représentations sociales, elles constituent une méthode particulièrement prisée par les chercheurs en développement régional, le développement ne se mesurant pas uniquement par l'évolution d'indicateurs socioéconomiques, mais aussi par la transformation des représentations des acteurs sociaux (Jodelet, 1997). Les réalités sociales résultent de la construction de ces mêmes acteurs qui donnent un sens à leur milieu (Savoie-Zjac, 2016). Par conséquent, les représentations permettent aux individus d'interpréter leur environnement suivant un processus dynamique, lequel est influencé par des facteurs tels que le degré de réceptivité aux stéréotypes du milieu, les valeurs sociales et l'appartenance sociale, les sentiments, les motivations, les connaissances et les souvenirs (Simard, 2003). Une telle approche, qui s'avère en parfaite adéquation avec les modèles de gérontologie environnementale et de renforcement des capacités, présente aussi l'avantage de ne pas dissocier l'objet et le sujet, chaque participant étant à la fois objet et sujet de l'action (Argoud \& Chazelle, 2011).

De manière plus explicite, notre cadre opératoire repose sur trois outils d'investigation, à savoir: des enquêtes de terrain, la réalisation d'entretiens semi-dirigés effectués auprès de différents intervenants œuvrant dans le domaine du vieillissement et la tenue de deux groupes de discussion (un par localité) réalisés auprès des personnes âgées.

\section{Stratégie de recrutement profil des participants et collecte des données}

L'approche par boule de neige a été utilisée comme méthode de recrutement, cette dernière permettant de cibler les participants en fonction des objectifs spécifiques de l'étude (Fortin \& Gagnon, 2016). Contactés en février 2017, les responsables des clubs d'âge d'or des deux localités à l'étude nous ont servi de personnes-ressources, nous dirigeant vers d'autres types d'intervenants œuvrant auprès des aînés. Ces intervenants nous ont fourni à leur tour les coordonnées d'aînés impliqués au sein de leur milieu respectif qui accepteraient de participer à un groupe de discussion.

Nous avons rencontré, en premier lieu, les représentants des deux DSL afin d'effectuer la recension des organismes et des services disponibles au sein du milieu. D'une durée moyenne de 90 minutes, les entrevues individuelles effectuées avec les intervenants se sont déroulées en mai et juin 2017. Elles ont permis de mieux cerner l'organisation des services locaux ainsi que les diverses initiatives mises de l'avant dans le but d'atténuer les effets liés à l'érosion des services de proximité. Quels sont les services et les actions mis en place pour les aînés? Comment s'effectue la prise en compte de leurs besoins en matière de services par les différents intervenants sociaux? Quels réseaux sont mobilisés? En quoi la distance et la dispersion représentent-elles des obstacles à la qualité de vie des personnes âgées? À quels services les personnes âgées envisagent-elles avoir recours et quels sont ceux dont elles pourraient éventuellement se passer? Voilà autant de questions qui ont été abordées dans le cadre de nos entretiens.

Pour les deux localités à l'étude, nous avons rencontré le représentant du DSL, trois fonctionnaires (deux à Robertville et un à Saint-Wilfred) du ministère du Développement social, le responsable du club de l'âge d'or et de la chorale paroissiale. À Robertville, nous avons interrogé la représentante des Filles d'Isabelle et un ancien membre du conseil d'administration de la coopérative. Deux membres du conseil d'administration du club de l'âge d'or ont participé à l'entrevue. À Saint-Wilfred, le responsable de l'accueil de jour, le curé du secteur pastoral, une infirmière praticienne et un représentant des Chevaliers de Colomb ont accepté de répondre à nos questions. Deux gestionnaires de foyers de soins ont été rencontrés ensemble à Robertville et un, sur une base individuelle, à Saint-Wilfred. Au total, 20 intervenants ont donc été sollicités, soit 10 à Robertville ( 2 hommes et 8 femmes) et 10 à Saint-Wilfred (4 hommes et 6 femmes). Nous avons effectué 17 entrevues, soit 9 à Saint-Wilfred et 8 à Robertville.

En ce qui concerne les groupes de discussion, ceux-ci se composaient de 11 personnes ( 7 femmes et 4 hommes) à Saint-Wilfred et de 7 personnes ( 4 femmes et 3 hommes) à Robertville. Nous avons donc réussi à obtenir une certaine représentativité, et ce, même si la sélection des répondants, en analyse qualitative, est effectuée en fonction de leur pertinence par rapport à l'analyse des données et non pour leur représentativité (LeCompte \& Schensuel, 2010). L'âge des participants variait entre 62 et 73 ans à Robertville et entre 65 et 86 ans à Saint-Wilfred. Deux personnes occupaient toujours un emploi à temps plein à Saint-Wilfred alors que toutes les autres étaient à la retraite, et ce, tant à Saint-Wilfred qu'à Robertville. Tous les participants pratiquaient une activité bénévole, que ce soit au niveau du club de l'âge d'or, de la localité, des loisirs, de l'entraide ou encore des services liés au maintien à domicile. Durant leur vie active, les participants cumulaient les fonctions et/ou titres suivants: gestionnaires de programmes, préposé aux bénéficiaires (2), commis de bureau (2), travailleur social, fonctionnaire pour le gouvernement fédéral, secrétaire, camionneur, employé du Canadien National (CN), boulanger, poissonnier et bûcheron. Trois personnes n'avaient jamais été sur le marché du travail. Dès lors, leurs profils socioprofessionnels étaient aussi particulièrement diversifiés. Tant les personnes âgées de Robertville que celles de Saint-Wilfred étaient natives de la région.

\section{Validité et analyse des données, portée et limites méthodologiques}

Nous nous sommes assuré de la fiabilité et de la validité des données recueillies en privilégiant une diversité des acteurs qui interviennent auprès des aînés (Gagnon, 2011). L'approche multiméthode a favorisé le recoupement des données, ce qui a également permis de les valider à l'interne (Denzin, 2008). Notre connaissance du terrain, la réalisation de prétests ${ }^{4}$, I'utilisation de données de sources différentes (entrevues auprès des représentants des DSL, données de Statistique Canada, sites web de Radio-Canada et de I'Acadie Nouvelle, etc.), la diversité des approches (enquêtes sur le terrain, entrevues, groupe de discussion) et la tenue de deux activités de dissémination au sein des localités à l'étude, soit en août 2018 dans le cas de Saint-Wilfred et en décembre 2018 dans celui de Robertville, s'inscrivent également dans la volonté d'assurer la validité des données à l'interne et à l'externe et de favoriser leur transférabilité.

En ce qui concerne l'analyse, nous avons effectué une transcription intégrale des propos que nous avons recueillis auprès des participants. II s'agissait de décrire leur expérience en organisant les données selon les objectifs de l'étude suivant la méthode d'émergence par le processus de comparaison constante (Morse \& Field, 1995) Après une relecture, nous avons procédé à un découpage en fonction des différents thèmes et sous-thèmes identifiés à partir de notre schéma d'entretien (Bardin, 2013). Nous avons comparé le contenu des entrevues entre elles de façon à faire ressortir les principaux éléments de similitude et de différenciation pour chacun des thèmes de notre grille d'entretien. Le croisement des données issues des entrevues et des deux focus group a été mis en perspective avec celles découlant de la littérature. Ce recoupement a favorisé un deuxième niveau d'analyse, ce qui a permis de projeter les données à travers le matériel recueilli (Durand \& Blais, 2009; Pinard, Potvin \& Rousseau, 2004; Delfosse \& Rouan, 2001). Quelques extraits de verbatim ont servi à illustrer les propos des différents participants. 
Certes, notre contribution comporte certaines limites. Par exemple, des entrevues individuelles réalisées auprès d'aînés auraient pu contribuer à consolider, voire à enrichir l'analyse. En effet, l'un des inconvénients des groupes de discussion est de susciter un certain consensus laissant peu de place aux voix discordantes. En outre, nos deux groupes de discussion étaient composés de personnes âgées actives et donc mobiles. Dès lors, nous sommes conscient que cette homogénéité ne reflète qu'une partie de la situation des aînés. Par ailleurs, force est de reconnaître que la caractéristique du sujet est susceptible d'influencer, du moins jusqu'à un certain point, la nature des résultats. Pour cette raison, ceux-ci doivent être interprétés comme un échantillon de la multiplicité des représentations sociales. Toutefois, en décelant certaines tendances fortes, les résultats obtenus sont indicatifs d'une certaine réalité. De plus, le fait d'avoir couplé les représentations sociales à une analyse de contenu contribue non seulement à la fiabilité, mais aussi à l'originalité de notre démonstration.

\section{RÉSULTATS}

\section{Le cas de Robertville}

Robertville est une localité du comté de Gloucester située à 12,4 kilomètres au nord-ouest de Bathurst (16 694 habitants en 2016). De 1981 à 2016, sa population est passée de 733 à 935 habitants, ce qui représente une augmentation de $27,6 \%$ (7,3\% pour la province). En comparaison avec la province (111\%), la localité de Robertville est davantage affectée par la gérontocroissance (117\%), mais surtout par le vieillissement par le haut (17,5 points de \% contre 9,8 points pour la province). Elle est aussi très fortement assujettie au vieillissement par le bas (-29,8 points de $\%$ contre $-18,8 \%$ pour la province) ainsi que par la juvénodécroissance (-70,5\% vs -37,9\%).

La fragilisation rurale affecte le milieu en raison du vieillissement de la population, de l'érosion des services de proximité et d'organismes communautaires et de la précarisation de l'économie au cours des 35 dernières années, cette dernière se traduisant par la perte d'emplois ainsi que par un niveau de revenu inférieur à la moyenne provinciale. Robertville se distingue néanmoins par le retour de jeunes au sein de la communauté. Selon un intervenant, la communauté se serait enrichie de 15 nouveaux résidents entre 2012 et 2017. Ce phénomène de retour se reflète également en suivant la cohorte des 0-24 ans en 1981, dont les effectifs font partie des 35-59 ans en 2016. De fait, Robertville affiche un gain net de 145 personnes entre 1981 et 2016 , soit une augmentation de $76,3 \%$ de ses effectifs (par rapport à $38,6 \%$ pour la province). La localité se caractérise aussi par le dynamisme de l'habitat ainsi que par la vitalité de la vie sociocommunautaire (club de l'âge d'or, plusieurs activités communautaires, nombreux organismes gravitant autour de l'église).

Bien que certains services, comme l'église, le bureau de poste et la cantine, soient toujours présents et utilisés par les personnes âgées, il n'en demeure pas moins qu'au cours des dernières années, Robertville et son environnement immédiat se sont caractérisés par l'effritement de leurs infrastructures de services, comme en témoigne d'ailleurs le tableau 1, ce qui s'est répercuté négativement sur la qualité de vie des aînés. Cet effritement s'est matérialisé par la fermeture de la coopérative, d'une station-service et d'un hôtel, la diminution des heures d'ouverture de la caisse populaire (fermeture définitive le 11 octobre 2019), le départ du curé résident, la vente du presbytère, la délocalisation de services religieux au profit de la localité voisine, le réaménagement des services de voirie, la perte de trois organismes communautaires (en l'occurrence les Filles d'Isabelle, les Chevaliers de Colomb et l'Association des vétérans) et de la chambre de relève que la Villa Sormany, une résidence pour personnes âgées, mettait à la disposition de la population de Robertville après un séjour à
I'hôpital, ainsi que par la suppression du service d'oto-rhino-laryngologie à l'hôpital de Bathurst.

Tableau 1. Services perdus ou ayant fait l'objet d'une restructuration dans les deux localités à l'étude et leur environnement immédiat

\begin{tabular}{ll} 
Robertville & Saint-Wilfred \\
\hline Fermeture de la coopérative & Fermeture de la coopérative \\
\hline Fermeture de la station-service & Fermeture de la station-service \\
\hline Fermeture d'un hôtel & Fermeture de deux dépanneurs \\
\hline $\begin{array}{l}\text { Diminution des heures de } \\
\text { service de la caisse populaire }\end{array}$ & Fermeture de la caisse populaire \\
\hline Départ du curé & Départ de religieuses \\
\hline Fermeture du presbytère & Fermeture du presbytère \\
\hline $\begin{array}{l}\text { Délocalisation des services } \\
\text { religieux }\end{array}$ & $\begin{array}{l}\text { Restructuration des services } \\
\text { religieux }\end{array}$ \\
\hline $\begin{array}{l}\text { Perte de trois organismes } \\
\text { communautaires }\end{array}$ & $\begin{array}{l}\text { Suppression d'un poste d'agent } \\
\text { de conservation de la faune }\end{array}$ \\
\hline $\begin{array}{l}\text { Perte de la chambre de relève } \\
\text { d'un foyer de soins }\end{array}$ & Fermeture du bureau de poste \\
\hline $\begin{array}{l}\text { Réaménagement des services } \\
\text { de voirie }\end{array}$ & Fermeture d'un restaurant \\
\hline $\begin{array}{l}\text { Suppression du service d'oto- } \\
\text { rhino-laryngologie (Bathurst) }\end{array}$ & $\begin{array}{l}\text { Fermeture d'un magasin } \\
\text { de plomberie }\end{array}$ \\
\hline
\end{tabular}

*Source : M. Simard

L'érosion des services de proximité a entraîné diverses conséquences sur la qualité de vie des personnes âgées et de la communauté. Parmi les aspects positifs, signalons: la mobilisation sociale des aînés à la suite du projet de fusion des directions générales de la Villa Sormany (Robertville) et du foyer Notre-Dame-de-Lourdes (Bathurst) et la stimulation de la capacité de résilience en particulier chez les personnes âgées de la nouvelle génération (65-74 ans), même lorsque couplée à un sentiment de résignation. À ce sujet, un intervenant déclare: «Il faut s'organiser. Nous n'avons pas le choix» (entrevue no 5). La restructuration des services a aussi entraîné l'émergence de stratégies organisationnelles, la consolidation du dynamisme communautaire et le renforcement de la solidarité territoriale. En ce qui concerne les aspects négatifs, soulignons un sentiment de découragement et d'incompréhension en particulier chez les aînés de la première génération ( 75 ans et plus): «Pour les aînés plus âgés, la perte de services est difficile à comprendre. Ce n'est pas facile à accepter » (entrevue no 5). L'inquiétude et l'insécurité concernant l'avenir des autres services de proximité, voire de la localité, ont également été soulevées. Certains acteurs ont évoqué l'effritement du tissu social entraînant son corollaire, l'accentuation de l'isolement social: "La coop, c'est une épicerie qui était beaucoup plus qu'une épicerie. C'était une fierté d'avoir la coop» (entrevue no 1), alors que d'autres ont mis l'accent sur les changements dans les habitudes de vie des aînés et la difficulté d'acceptation et d'adaptation en particulier chez les aînés de la première génération: «Le fait d'avoir un prêtre directement là, pour beaucoup des aînés, a été une grosse perte. II y en a qui ont eu de la difficulté à accepter ça» (entrevue no 2). Le mode de gouvernance des caisses populaires acadiennes a aussi été remis en question: "Les décisions semblent avoir été prises d'avance» (entrevue no 4). De leur côté, les aînés ont déploré les difficultés concernant l'accessibilité des services, en particulier pour ceux qui ne possèdent pas de voiture ou dont les enfants sont éloignés. L'érosion des services locaux oblige en effet les personnes âgées à se déplacer sur de plus longues distances: 
« II n'y a plus de magasin et d'épicerie. On est obligé de se rendre à Beresford. La caisse populaire ferme à $1 \mathrm{~h} 00$ maintenant et peut-être qu'elle va fermer complètement. On va être obligé d'aller soit à PetitRocher ou à Beresford pour faire nos choses. C'est un des désavantages » (groupe de discussion).

La faiblesse de l'attractivité de la localité, un sentiment d'abandon, voire de tristesse et l'allongement du délai pour obtenir des services figurent également parmi les conséquences qui ont été mentionnées.

Par ailleurs, la dissolution progressive de l'infrastructure de services a eu pour effet de développer la capacité de résilience des aînés. De fait, les intervenants communautaires ont identifié diverses stratégies organisationnelles déployées par les aînés, lesquelles ont été corroborées par ces derniers lors des deux groupes de discussion. Parmi ces stratégies soulignons: la planification à l'avance de menus (depuis la fermeture de la coopérative), la sollicitation des membres de la famille, d'amis, de voisins ou de bénévoles pour faciliter l'accès aux services (ramener des vivres, aller faire l'épicerie à Bathurst ou à Beresford, etc.), la mise à disposition d'enveloppes et de formulaires à l'entrée de l'église pour les aînés qui souhaitent faire célébrer une messe, la fréquentation de la caisse populaire plus tôt le matin ou de celles de Petit-Rocher ou de Beresford offrant plus de services, la familiarisation des aînés de la nouvelle génération avec les services offerts en ligne par la caisse populaire, l'organisation, sur une base ponctuelle, de manifestations et l'utilisation de taxis pour effectuer des achats ou pour accéder à des services qui ne sont pas disponibles au sein de la localité.

\section{Le cas de Saint-Wilfred}

Saint-Wilfred est situé dans le comté de Northumberland à 41 kilomètres de Miramichi (17 537 habitants). Au recensement de 2016, sa population s'établissait à 765 habitants comparativement à 1155 en 1981, une diminution de 33,8\%. La localité de Saint-Wilfred est fortement affectée par la gérontocroissance (augmentation de $156 \%$ du nombre de personnes âgées entre 1981 et 2016 par rapport à $111 \%$ pour la province), le vieillissement par le haut (11,4 points de \% contre 9,8 points pour la province), la juvénodécroissance (diminution de $73,9 \%$ par rapport $37,8 \%$ pour la province), le vieillissement par le bas (diminution de 36,2 points de \% par rapport à -18,8 points pour la province) et par l'émigration des jeunes. De fait, en suivant la cohorte des 0-24 ans en 1981, dont les effectifs font partie des 35-59 ans en 2016, Saint-Wilfred affiche une perte nette de 385 personnes, soit une diminution de 55,8\% de ses effectifs (par rapport à une augmentation de $38,6 \%$ pour la province).

Contrairement à la localité de Robertville, celle de Saint-Wilfred se caractérise par la très forte sous-scolarisation de sa population ${ }^{5}$. En effet, près des deux tiers de celle-ci n'a pas atteint le seuil de la $9 \mathrm{e}$ année en 2016 par rapport à $22 \%$ pour la province. Par ailleurs, au cours de la période 1981-2016, la situation économique de Saint-Wilfred s'est améliorée en raison de la relative proximité de cette localité par rapport à Miramichi et à Neguac. Cette amélioration, attribuable à la meilleure santé économique de ces deux localités, s'est traduite par une augmentation de son taux d'activité, une diminution du taux de chômage, la création de 25 emplois ainsi que par le quadruplement du revenu moyen des familles.

La localité de Saint-Wilfred semble avoir été encore plus affectée que celle de Robertville par l'érosion de ses services de proximité au cours des dernières années. Comme l'indique le tableau 1, cette érosion s'est matérialisée par la perte de tous ses services (à l'excep- tion du club de l'âge d'or) et, plus spécifiquement, par la fermeture du bureau de poste et de la caisse populaire (Lavillette), la vente du presbytère, le départ des Sœurs salésiennes qui étaient fortement impliquées au sein de la communauté, la fermeture de la coopérative de Tabusintac, la suppression d'un poste d'agent de conservation de la faune et la restructuration des services religieux. Deux intervenants ont bien mis en évidence la forte érosion de l'infrastructure de services, dont a souffert la localité de Saint-Wilfred:

«Quand j'ai déménagé dans le coin, on avait beaucoup d'entreprises. On avait une caisse populaire à plein temps avec tous les services. On avait un bureau de poste. On avait un presbytère. On avait un petit restaurant. On avait une épicerie. On avait une station d'essence. On avait un couvent avec des religieuses. On avait une école avec tous les services et un magasin de plomberie. Maintenant, on a des lumières dans les rues. Le presbytère, le couvent, le restaurant, le magasin, l'essence, la caisse, le bureau de poste sont partis. L'école reste, mais c'est fragile. L'église est encore ouverte. Mais je ne sais pas pour combien de temps, probablement pas longtemps » (entrevue no 11).

«II y a 15 ans passés à Saint-Wilfred, il y avait un petit dépanneur pas loin où tu pouvais avoir l'essentiel. II y avait une grosse épicerie près de l'église à Lagacéville. II y avait un autre gros magasin à Lavillette. Ils ont tous fermé, parce que ces petits dépanneurs-là ne pouvaient plus subsister » (entrevue no 12).

À l'instar de Robertville, l'érosion des services de proximité s'est répercutée à la fois positivement et négativement sur la qualité de vie des aînés. En ce qui a trait aux impacts positifs, elle a suscité une mobilisation des aînés, quoique ponctuelle, à la suite de la fermeture du presbytère, en vue de sauvegarder l'église et de mettre fin au projet de transfert des services ambulanciers de l'hôpital de Miramichi vers celui de Tracadie. L'effritement des services a aussi contribué à stimuler la capacité de résilience des aînés: "Les personnes âgées n'ont pas le choix de s'adapter aux changements puisque les choses changent toujours. Je pense quand même qu'elles s'adaptent» (entrevue no 14). Enfin, l'érosion des services de proximité a favorisé l'émergence de stratégies organisationnelles et la consolidation du dynamisme communautaire.

Les impacts négatifs se sont toutefois avérés nombreux. Parmi ceuxci, mentionnons un sentiment de frustration, voire de privation:

«Les aînés se sentent privés des choses qu'ils ont peutêtre perdues » (entrevue no 11).

«Le bureau de poste a été un coup dur à oublier, parce qu'il y avait du monde qui était un peu frustré. II y avait une seule job fédérale dans la place et on l'a perdue. C'est peut-être parce que l'on ne vaut pas grand-chose sur la carte électorale » (entrevue no 17). 
«La caisse et le bureau de poste ont été de grosses pertes pour nous » (groupe de discussion).

La dissolution de l'armature de services a suscité de l'inquiétude quant à l'avenir du seul service existant, à savoir le club de l'âge d'or: "Les personnes âgées ont peur que tous les services s'en aillent. Elles ont de la misère quand il y a des fermetures » (entrevue no 12). Les intervenants sociaux ont également été à même de constater l'effritement du tissu social entraînant son corollaire, l'accentuation de l'isolement: «Quand tu arrivais à la caisse populaire, la personne qui t'accueillait te connaissait par ton nom [...]. Donc, c'est ce sentiment de proximité qui régnait. Lorsque tu perds ce sentiment, ça crée un grand vide» (entrevue no 16). La fermeture de l'épicerie a aussi contribué à réduire le niveau d'autonomie de certaines personnes âgées, le phénomène étant exacerbé en l'absence d'un service de transport collectif:
«C'est un inconvénient qu'il n'y ait plus d'épicerie. II faut conduire jusqu'à Neguac pour aller au magasin. J'ai une auto. Mais je ne conduis pas loin. Ça fait qu'il faut que j'aille avec quelqu'un d'autre» (groupe de discussion).

«On n'a pas de taxi. Ça me manque beaucoup. Je ne peux pas conduire. II n'y a pas d'autobus non plus. On n'a rien de ça. Pour dire la franche vérité, on n'a rien. Les magasins sont un peu loin. Ça me dérange, parce que je n'ai pas de moyens de transport » (groupe de discussion).

Tableau 2. Synthèse des aspects positifs et négatifs liés à la dissolution des services de proximité au sein des localités de Robertville et de Saint-Wilfred et mesures organisationnelles déployées par les aînés dans le but d'en réduire les effets)

Conséquences liées à la perte de services de proximité

\section{Robertville}

\section{Saint-Wilfred}

\section{Aspects positifs}

- Mobilisation sociale des aînés à la suite du projet de fusion des directions générales de la Villa Sormany (Robertville) et du foyer Notre-Dame-de-Lourdes (Bathurst);

- Stimulation de la capacité de résilience en particulier chez les aînés de la nouvelle génération (65-74 ans) même lorsque couplée à un sentiment de résignation;

- Émergence de stratégies organisationnelles;

- Consolidation du dynamisme communautaire;

- Renforcement de la solidarité territoriale.
- Mobilisation sociale des aînés à la suite de la fermeture du presbytère, en vue de sauvegarder l'église et de mettre fin au projet de transfert des services ambulanciers de l'hôpital de Miramichi vers celui de Tracadie;

- Stimulation de la capacité de résilience couplée à un sentiment de résignation;

- Émergence de stratégies organisationnelles;

- Consolidation du dynamisme communautaire.

\section{Aspects négatifs}

- Sentiments de découragement et d'incompréhension en particulier chez les aînés de la première génération (75 ans et plus);

- Inquiétude et insécurité concernant l'avenir des autres services de proximité, voire de la localité;

- Effritement du tissu social

- Changements dans les habitudes de vie des aînés;

- Difficulté d'adaptation en particulier chez les aînés de la première génération;

- Questionnement des aînés quant au mode de gouvernance de certains organismes (en particulier des caisses populaires)

- Difficultés concernant l'accessibilité des services en particulier pour les aînés qui ne possèdent pas de voiture ou dont les enfants sont éloignés;

- Déplacements sur de plus longues distances des aînés pour obtenir des services jadis disponibles dans un rayon de moins de $10 \mathrm{~km}$;

- Faible attractivité de la localité;

- Allongement des délais pour obtenir des services;

- Sentiment d'abandon, voire de tristesse;

- Exode rural (en raison notamment de la fermeture de la coopérative).

Stratégies organisationnelles déployées par les aînés

\section{Robertville}

- Planification à l'avance de menus (depuis la fermeture de la coopérative);

- Sollicitation des membres de la famille, d'amis, de voisins ou de bénévoles pour faciliter l'accès aux services (ramener des vivres, aller faire l'épicerie à Bathurst ou à Beresford, etc.);

- Mise à disposition d'enveloppes et de formulaires à l'entrée de l'église pour les aînés qui souhaitent faire célébrer une messe;

- Fréquentation de la caisse populaire plus tôt le matin ou de celles de Petit-Rocher ou de Beresford offrant plus de services;

- Familiarisation des aînés de la nouvelle génération avec les services d'AccèsD;

- Organisation, sur une base ponctuelle, de manifestations;

- Utilisation de taxis (dans le cas d'aînés qui ne possèdent pas de voiture) pour effectuer des achats ou accéder à des services qui ne sont pas disponibles au sein de la localité.
- Sentiments de frustration et de privation;

- Inquiétude quant à l'avenir du seul service existant : le club de l'âge d'or;

- Effritement du tissu social entraînant son corollaire, l'accentuation de l'isolement social;

- Changements dans les habitudes de vie des aînés;

- Difficultés d'acceptation et d'adaptation en particulier chez les aînés

de la première génération;

- Cessation de la fréquentation de certains services, voire éclatement de rébellion;

- Perte de repères. 
La perte de services a forcé les aînés à modifier leurs habitudes, ce que certains vivent comme une forme de déracinement: «Ça l'a été un problème quand la coop a fermé, car j'allais toujours magasiner là. Quand ç'a fermé, j'ai été obligé d'aller ailleurs. Ce n'est pas loin pour nous d'aller à la ville. Mais ce n'est pas notre monde. Ce n'est pas notre région. Quand on allait à la coop, on voyait notre monde» (groupe de discussion).

Les personnes âgées de la première génération semblent avoir eu plus de difficultés à accepter et à s'adapter à la restructuration des services: "Quand on a fusionné les quatre paroisses, nous n'avons eu qu'une seule messe par semaine. On n'a plus les mêmes services. Il faut partager. Ça l'a pris du temps avant que les aînés acceptent ça» (entrevue no 15). Les difficultés d'adaptation ont été telles que des aînés auraient cessé de fréquenter certains services:

« Ma belle-mère, quand ils ont démoli le presbytère, était fâchée au point d'aller à l'église ailleurs, parce qu'elle ne pouvait pas comprendre pourquoi ils détruisaient le presbytère. II aurait dû avoir une autre utilité pour l'édifice et ils n'auraient pas dû le démolir » (entrevue no 11).

Au final, il s'en serait suivi une perte de repères:

« Il y a beaucoup de personnes âgées qui ont mal pris ça, la fermeture de la coop, parce qu'elles allaient toujours magasiner là. Elles étaient membres et elles ne voulaient pas aller ailleurs » (entrevue no 17).

«Le départ des religieuses, ça a été probablement dur pour elles, parce qu'elles étaient très impliquées dans la communauté. Elles faisaient des camps durant l'été avec les jeunes. Ça l'a été abandonné » (entrevue no 14).

Suite à la dissolution des services de proximité, les aînés auraient fait appel aux membres de leur famille et de leur entourage pour l'obtention de biens et de services. Parce qu'elles ne peuvent plus se rendre à la caisse populaire de Lagacéville, des personnes âgées auraient signé des procurations afin que leurs enfants puissent effectuer des transactions avec leur carte de crédit. Même si Saint-Wilfred n'a jamais disposé d'infrastructures sportives à destination des aînés, ceux-ci, en l'absence de tels équipements, choisiraient de faire leur promenade à pied ou en vélo très tôt le matin afin d'éviter le trafic. D'autres marcheraient face aux voitures pour assurer leur sécurité. D'autres encore auraient fait l'acquisition de balançoires. Enfin, des aînés, propriétaires de lots à bois ou de camps, iraient marcher sur leurs terres, autant de comportements qui témoignent de leur capacité d'agir.

\section{DISCUSSION}

Province à dominante rurale, le Nouveau-Brunswick se caractérise par la faiblesse de sa structure de peuplement, laquelle se matérialise par la dispersion de la population, la forte proportion de localités de petite taille démographique ainsi que par leur éloignement par rapport aux villes. Ces caractéristiques ont des impacts sur la nature et l'offre de services de proximité (Dugas, 2016; 1996). Outre les spécificités relatives à la structure spatiale, les changements dans les habitudes de consommation, l'avènement des nouvelles technologies d'information et de communication, le déclin de la pratique religieuse et du nombre de prêtres, les décisions administratives et gouvernementales et le vieillissement de la population sont autant de facteurs qui contribuent à l'érosion des services en milieu rural (Simard, 2006). Cette dernière est susceptible de se répercuter négativement sur la qualité de vie des personnes âgées, et en particulier sur celles de la première génération, c'est-à-dire les plus avancées en âge, souvent moins mobiles et moins enclines aux changements (Ottman \& Maragoudaki, 2015; Vandersmissen, 2012; Wild, Wiles \& Allen, 2013). Les conséquences liées à l'effritement des services ont aussi tendance à se manifester avec une plus grande acuité en milieu rural fragile, dont l'infrastructure de services est déjà ténue (Simard, 2003). C'est le cas de Robertville et de Saint-Wilfred, où les inconvénients liés à la dissolution des services de proximité apparaissent, tant pour les aînés que pour les intervenants sociaux, beaucoup plus nombreux que les avantages.

La perte de services de proximité a entraîné au sein des deux localités à l'étude, différents impacts négatifs, dont un sentiment d'incompréhension, de la frustration, du découragement, de l'inquiétude et de l'insécurité quant à l'avenir des autres services. Cette érosion a également provoqué l'effritement du tissu social, des changements dans les habitudes de vie ainsi que des difficultés d'adaptation. Les intervenants et les aînés de Robertville, en dépit de la proximité de cette localité par rapport à Bathurst ont, en comparaison avec leurs homologues de Saint-Wilfred, identifié un plus grand nombre d'inconvénients liés à l'érosion de l'infrastructure de services. Parmi ceux-ci, rappelons les difficultés concernant l'accès aux services pour les aînés qui ne possèdent pas de voiture ou dont les enfants sont éloignés, le déplacement sur de plus longues distances et donc, l'allongement du délai pour obtenir des services, l'affaiblissement de l'attractivité de la localité ainsi que l'exode rural. À Saint-Wilfred, la perte de services semble engendrer une certaine forme de carence chez les personnes que nous avons interrogées. À preuve, des intervenants ont évoqué que les aînés n'auraient d'autre choix que de s'abstenir de consommer des services advenant leur disparition. Mais la perte de services n'a pas que des inconvénients. Elle entraîne le déploiement de stratégies organisationnelles, dont certaines vont bien au-delà du cadre local, témoignant ainsi de l'ancrage territorial des aînés à l'égard de leur milieu de vie tout en s'inscrivant dans le sens de la promotion d'un vieillissement actif (Klein, 2018; Caradec, 2012). Dès lors, l'érosion des services de proximité contribue au renforcement des capacités des aînés (Bédard \& Beaulieu, 2015; Garon et al., 2012).

Tant à Robertville qu'à Saint-Wilfred, ce renforcement se traduit par la stimulation d'une certaine forme de résilience et d'empowerment, l'émergence d'une mobilisation ponctuelle et la consolidation du dynamisme communautaire. Les stratégies déployées par les aînés sont d'autant plus louables qu'elles sont mises de l'avant par des personnes vulnérables et fragiles, renforçant ainsi le potentiel d'empowerment (Pelchat, 2010). Par ailleurs, bien que réelle, la capacité d'agir des aînés n'est pas porteuse d'un projet individuel ou collectif ni de progrès social (Pelchat, 2010; LeBossé, 1996). On peut aussi se demander jusqu'à quel point ces stratégies sont en mesure d'influencer le comportement des autres, l'une des principales caractéristiques de l'empowerment (Nincas, 2002). Elles n'ont pas non plus pour finalité «l'exercice d'un plus grand contrôle sur ce qui est important pour soi ou sa collectivité »(LeBossé, 2003: 39). Bien qu'elles répondent à un besoin, elles ne favorisent pas une transformation de l'environnement social des aînés (Lemay, 2007). Pour ces raisons, leur contribution en matière d'empowerment s'avère limitée.

En ce qui concerne la résilience, les stratégies organisationnelles mises en place par les intervenants sociaux et les aînés témoignent de leur volonté à affronter certaines difficultés qui, bien qu'elles soient hors de leur contrôle, leur permettent de s'en sortir en étant mieux adaptés (Luc, 2010). Sans constituer un vecteur de change- 
ment, le déploiement de ces stratégies permet aux intervenants et aux aînés de consolider leur rôle d'acteur social en plus de contribuer à atténuer les effets liés à la perte de services (Alberio, 2015). Par ailleurs, leurs impacts, en ce qui a trait à l'amélioration de leur qualité de vie ainsi que sur la dynamique de développement local, apparaissent plutôt modestes. Elles ne s'inscrivent pas dans une optique de durabilité, mais visent plutôt à satisfaire des besoins de base (Musson, 2013). Par conséquent, ces stratégies, qui prennent davantage la forme de mesures d'adaptation, ne peuvent pas être considérées comme des innovations sociales au sens où l'entend le CRISES (Argoud, 2010). En outre, ces dernières ne sont pas porteuses de transformation sociale (Klein et al., 2016). Elles ne sont pas issues d'une mobilisation générale et massive des forces endogènes et exogènes du territoire ni d'une perspective de redynamisation de celui-ci, autant de caractéristiques associées à la résilience territoriale (Tanguy \& Charreyron-Perchet, 2013-2014; Rallet, 2007). Dès lors, elles n'ont pas d'incidences significatives en matière de développement (Jean, 2015).

D'autre part, pour qu'elles puissent être considérées comme des innovations sociales et ainsi contribuer au renforcement des capacités, les initiatives mises en place par les aînés devraient favoriser l'émergence de nouvelles formes de prestation de services, la concertation d'acteurs provenant de milieux différents ainsi que la formation d'alliances et de partenariats inédits, et non s'inscrire dans une situation d'obligation (Klein et al., 2019; Klein \& Champagne, 2011). L'amélioration de la qualité de vie des personnes âgées résidant en milieu rural fragile passe incontestablement par le renforcement de l'infrastructure de services (et non par leur dissolution), une mesure qui outrepasse la responsabilité et les compétences des intervenants sociaux et des aînés. Une telle mesure, qui relève davantage de l'État, nécessite la mise en œuvre d'une politique du vieillissement arrimée à une stratégie d'aménagement du territoire (Lord \& Piché, 2018; Chapon et al., 2013; Hodge, 2008). L'élaboration d'une telle stratégie doit s'effectuer dans le souci de favoriser une certaine forme d'équité territoriale tout en prenant en considération les comportements et les besoins des personnes âgées, mais aussi les caractéristiques géographiques particulières au Nouveau-Brunswick, dont la petite taille démographique des localités, la distance par rapport aux villes, la forte dispersion de la population et la dualité linguistique.

\section{BIBLIOGRAPHIE}

Alberio, M. (2015). Les initiatives locales et les défis des acteurs du milieu face aux coupes et aux changements actuels. Revue Organisations \& Territoires 3: 53-60.

Argoud, D. (2010). Le pouvoir gris à l'épreuve du territoire. Gérontologie et Société 132: 63-76.

Argoud, D. (2017). Territoires et vieillissement: vers la fin de la politique vieillesse? Lien social et Politiques 79: 17-34.

Argoud, D. \& M. Chazelle (2011). Politique territoriale de la vieillesse. L'exemple du CLIC du Diois (Drôme). Le sociographe 35: 33-43.

Atkinson, A.B. (2016). Inégalités. Paris: Éditions du Seuil.

Aubry, B. \& J.-F. Léger (2015). L'inégalité économique des territoires. Les écarts continuent de se creuser. Population \& Avenir, 722: 4-7.

Badie, B. \& D. Vidal (2017). Un monde d'inégalités. L'état du monde 2016. Paris: La Découverte.

Bardin, L. (2013). L'analyse de contenu. Paris: Presses universitaires de France.

Bédard, M.-È. \& M. Beaulieu (2015). La défense des droits des aînés au Québec: protection et empowerment. Le Sociographe 50: 33-42.
Besançon, E. \& N. Chochoy (2019). Mesurer l'impact de l'innovation sociale: quelques perspectives en dehors de la théorie du changement? Revue internationale de l'économie sociale 352: 42-58.

Besançon, E., N. Chochoy \& T. Guyon (2013). L'innovation sociale. Principes et fondements d'un concept. Paris: L'Harmattan.

Bouchard, M.J. (2011). L'économie sociale: vecteur d'innovation. L'expérience du Québec. Québec: Presses de l'Université du Québec.

Bret, B. (2009). Interpréter les inégalités socio-spatiales à la lumière de la Théorie de la Justice de John Rawls. Annales de géographie 665-666: 16-34.

Caradec, V. (2012). Sociologie de la vieillesse et $d u$ vieillissement. Paris: Armand Colin.

Chapon, P.-M. (2013). Bâtir une ville pour tous les âges. Paris: La Documentation française.

Chapon, P.-M., M. Codacioni, O. Morlet, C. Vaudry \& A. Sultan (2013). Adapter les villes au vieillissement. Enjeux d'aménagement et de gouvernance. Paris: Centre d'analyse stratégique.

Cyrulnik, B. (1998). Ces enfants qui tiennent le coup. Revigny-sur-Ornain: Hommes et Perspectives.

Davis, C.A. (2008). Le renforcement des capacités: I'habilitation des gens et des institutions, Rapport annuel. New York: Programme des Nations unies pour le développement.

Delfosse, M. S. \& G. Rouan (2001). Les méthodes qualitatives en psychologie. Paris: Dunod.

Denzin, N \& Y. Lincoln (2008). The Landscape of Qualitative Research, 3d ed. Thousand Oaks: Sage.

Dugas, C. (2016). Dimensions politiques et structurelles de la marginalisation en milieu rural. Communication 53 e colloque de l'ASRDLF, 7-9 juillet.

Dugas, C. (1996). L'espace rural canadien. Sainte-Foy: Presses de I'Université du Québec.

Dugas, C. (1994). Les disparités canadiennes. Norois 163: 367-384.

Dumont, G.-F. (2012). Diagnostic et gouvernance des territoires. Concepts, méthode, application. Paris: Armand Colin.

Dumont, G.-F. (2018). Géographie des populations. Concepts, dynamiques, prospectives. Paris: Armand Colin.

Durant, C. \& A. Blais (2009). La mesure, dans B. Gauthier (éd.), Recherche sociale. De la problématique à la collecte des données. Québec: Presses de l'Université du Québec, 227-250.

El-Batal, K. \& A. Joyal (2015). La Politique nationale de la ruralité québécoise relève-t-elle d'une gouvernance stratégique territoriale? Cahiers de géographie 167: 189-207.

Fortin, M.-F. \& J. Gagnon (2016). Fondements et étapes du processus de recherche. Méthodes quantitatives et qualitatives. Montréal: Chenelière Éducation.

Gagnon, Y.-C. (2011). L'étude de cas comme méthode de recherche. Québec: Presses de l'Université du Québec.

Garon, S., M. Beaulieu, A. Veil, M. Paris \& C. Bigonesse (2012). L'expérience québécoise du programme «Villes amies des aînés 》 de I'OMS: I'implantation dans sept projets pilotes, dans J.P. Viriot-Durandal, C. Pihet \& P.-M. Chapon (éd.), Les défis territoriaux du vieillissement. Paris: La Documentation française, 69-88.

Garon, S., M. Paris, M. Beaulieu \& A. Laliberté (2014). Collaborative partnership in age-friendly cities: Two case studies from Quebec, Canada. Journal of Aging \& Social Policy 1-2: 73-87. 
Grenier, J. \& M. Simard (2011). Une éthique pour l'autonomie. Travail social et empowerment au Québec. Le Sociographe 35: 75-80.

Gucher, C. (2013). Le vieillissement des populations et des territoires au prisme d'une ruralité transformée. Gérontologie et société 146: 1120.

Guedeney, A. (1998). Les déterminants précoces de la résilience, dans B. Cyrulnik (ed.), Ces enfants qui tiennent le coup. Revigny-sur-Ornain : Hommes et Perspectives, 13-26.

Hodge, G. (2008). The Geography of Aging; Preparing Communities for the Surge in Seniors. Montréal: McGill-Queen's University Press.

Kending, H. (2003). Directions in Environmental Gerontology: a multidisciplinary field. The Gerontologist 5: 611-615.

Klein J.-L., J. Boucher, A. Camus, C. Champagne \& Y. Noiseux (2019). Trajectoires d'innovation. Des émergences à la reconnaissance. Québec: Presses de l'Université du Québec.

Klein, J.-L., A. Camus, C. Champagne \& C. Roy (2016). La transformation sociale par l'innovation sociale. Québec: Presses de l'Université du Québec.

Klein, A. (2018). Vieillissement, innovation et territoire. Le cas de l'île de la Réunion. Paris: L'Harmattan.

Klein, J.-L. (2017). L'innovation sociale au cœur de l'analyse de la transformation sociale. La programmation du CRISES 2014-2020. Montréal: Cahiers du CRISES.

Klein, J.-L. \& C. Champagne (2011). Initiatives locales et lutte contre la pauvreté et l'exclusion. Québec: Presses de l'Université du Québec.

Klein, J.-L., J.-M. Fontan, D. Harrisson \& B. Lévesque (2009). L'innovation sociale au Québec: un système d'innovation fondé sur la concertation. Montréal: Cahiers du CRISES.

Iwarsson, S. (2005). A long-term perspective on person-environment fit and ADL dependance among older Swedish adults. The Gerontologist 3 : 327-336.

Jean, B. (2012). Les territoires ruraux au Québec: vers un modèle de développement territorial durable. Revue d'Économie Régionale \& Urbaine 4:649-671.

Jean, B. (2015). Le développement territorial durable: une nouvelle approche de développement rural. Desenvolvimento e Meio Ambiente 33: 115-127.

Jodelet, D. (1997). Les représentations sociales. Paris: Presses universitaires de France.

Lawton, P. (1986). Environment and ageing. Albany: Center for Study of Ageing.

Lawton, P. (1998). Environment and ageing: Theory revisited, dans R.J. Scheidt \& P. Windley (éd.), Environment and aging Theory: A forces on housing. London: Greenwood Press, 1-31.

LeBossé, Y. (2003). De l'« habilitation » au « pouvoir d'agir » : vers une appréhension plus circonscrite de la notion d'empowerment. Nouvelles pratiques sociales $2: 30-51$.

LeBossé, Y. (1996). Empowerment et pratiques sociales: illustration du potentiel d'une utopie prise au sérieux. Nouvelles pratiques sociales 1: 127-145.

Lecompte, M.D. \& J.J Schensul (2010). Designing and conducting ethnographic research, vol. 1. Walnut Creek: Rowman Altamira.

Lemay, L. (2007). L'intervention en soutien à l'empowerment: du discours à la réalité. La question occultée du pouvoir entre acteurs au sein des pratiques d'aide. Nouvelles pratiques sociales 1: 165-180.
Lesourd, M. (2003). Nouvelles technologies, nouvelles inégalités? Les NTIC et les fractures sociospatiales, dans J.-M Amat-Rose (éd.), L'Afrique, vulnérabilité et défis. Paris: Éditions du Temps, 392-413.

Lord, S. \& D. Piché (2018). Vieillissement et aménagement. Perspectives plurielles. Montréal: Presses de l'Université de Montréal.

Luc, É. (2010). Le leadership partagé. Montréal: Presses de l'Université de Montréal.

Manciaux, M. (2004). De la répétition des traumatismes à l'ouverture vers d'autres possibles. Conférence prononcée dans le cadre du colloque Résilience et intervention clinique: espoir ou utopie? Montréal : Hôpital Ste-Justine.

Meloche, J.-P. (2012). Impacts de la décentralisation fiscale et de la déconcentration sur les disparités régionales: le cas des provinces canadiennes. Revue canadienne des sciences régionales 1: 19-28.

Morse, J. \& P. Field (1995). Qualitative research methods for health professionals. Thousand Oaks: Sage Publications.

Musson, A. (2013). Attractivité durable et résilience des territoires, dans É. Laurent (ed.), Vers l'égalité des territoires. Dynamiques, mesures, politiques. Paris: La Documentation française, 315-333.

Ninacs, W.A. (2012). Empowerment et intervention. Développement de la capacité d'agir et de la solidarité. Québec: Presses de l'Université Laval.

Ninacs, W.A. (2002). Le pouvoir dans la participation au développement local, dans M. Tremblay, P.-A Tremblay \& S. Trembay (ed.). Développement local, économie sociale et démocratie. Sainte-Foy: Presses de l'Université du Québec, 25-50.

Ottmann, G \& M. Maragoudaki (2015). Fostering resilience later in life: a narrative approach involving people facing disabling circumstances, carers and members of minority groups. Ageing and Society 10: 2071-2099.

Pech, M., Y. Léon \& P. Dupraz (2001). Soutien public à l'agriculture et au développement rural: l'équité introuvable? Économie rurale 262 : 109-116.

Pelchat, Y. (2010). L'appel à la participation: une vision privatisée de l'inégalité?. Nouvelles pratiques sociales 2: 114-129.

Piketty, T. (2019). Le capital au XXle siècle. Paris: Éditions du Seuil.

Pinard, R., P. Potvin \& R. Rousseau (2004). Le choix d'une approche méthodologique mixte de recherche en éducation. Recherches qualitatives 24: 58-82.

Rallet, A. (2007). Commentaires, dans A. Mollard, E. Sueboua \& M. Hirczak (éd.), Territoires et enjeux du développement régional. Versailles: Quae, 2007, 80-82.

Savoie-Zajc, L. (2016). L'entrevue semi-dirigée, dans B. Gauthier \& I. Bourgeois (éd.), Recherche sociale. De la problématique à la collecte des données. Québec: Presses de l'Université du Québec, 327-364.

Scheidt, R. J. et B. Schwarz (2013). Environmental Gerontology. What now? Abington: Routledge.

Simard, M. (2003). La fragilité de l'espace rural québécois. Le cas des petites localités du Bas-Saint-Laurent: enjeux et perspectives d'avenir. Rimouski: Thèse de doctorat, Université du Québec à Rimouski.

Simard, M. (2018). Le JAL: un demi-siècle de luttes et de débats. Bilan et perspectives nouvelles d'une expérience de développement communautaire en milieu rural fragile. Rimouski: GRIDEQ.

Simard, M. (2019). Les enjeux et les défis du vieillissement en milieu minoritaire francophone: une analyse sous l'angle des représentations sociales des acteurs. Le cas de Tracadie au Nouveau-Brunswick. Minorités linguistiques et société 11: 3-28. 
Simard, M. (2016). Les petites localités rurales de l'Atlantique: une analyse sous l'angle de la fragilité et de la fragilisation territoriale. Revue de l'Université de Moncton 1: 101-135.

Simard, M. (2006). Les services de proximité aux populations rurales: rapport-synthèse sur les connaissances et les enseignements des initiatives novatrices. Rapport no 3. Rimouski: Chaire de recherche du Canada en développement rural.

Simard, M. (2015). Quelques jalons en vue de l'élaboration d'une politique territoriale du vieillissement au Nouveau-Brunswick. Vie et vieillissement $3: 33-41$.

Simard, M., T. Diallo \& S. Tremblay (2014). Théories et pratiques en développement territorial: des grands projets aux initiatives locales et vice versa. Revue Organisations \& Territoires 3: 5-14.

Simard, M., S. Dupuis-Blanchard, O. Gould, L. Villalon, S. Éthier \& C. Gibbons (2015). L'influence du contexte sociolinguistique minoritaire sur le maintien à domicile des aînés en milieu rural dévitalisé: le cas d'Acadieville au Nouveau-Brunswick. Revue canadienne du vieillissement 2: 194-206.

Tanguy, J.-M. \& A. Charreyron-Perchet (2013-2014). La résilience territoriale: un premier diagnostic. Annales des Mines - Responsabilité et environnement $72:$ 32-36.

Thiesse, J.-F. (1994). L'équité spatiale, dans J.P. Auray, A. Bailly \& P.H. Derycke (éd.), Encyclopédie d'économie spatiale: concepts, comportements, organisations. Paris: Économica, 225-232.

Tremblay, D.-G., J.-L. Klein \& J.-M. Fontan (2016). Initiatives locales et développement socioterritorial. Québec: Presses de I'Université du Québec.

Vandersmissen, M.-H. (2012). Mobilité et espaces d'activité des 65 ans et plus dans la région urbaine de Québec, dans P. Negron-Poblète \& A.-M. Séguin (éd.), Vieillissement et enjeux d'aménagement. Regards à différentes échelles. Québec: Presses de I'Université du Québec, 31-65.

Von Bardelehen, E. (2016). Empowerment: le grand détournement de fond. Le Devoir 203: A-7.

Wahl, H. W. \& G.D. Weisman (2003). Environmental Gerontology at the Beginning of the New Millennium: Reflections on Its Historical, Empirical, and Theoretical Development. The Gerontologist 5: 616627.

Wild, K., J.L. Wiles \& R.E.S. Allen (2013). Resilience: thoughts on the value of the concept for critical gerontology. Ageing and Society, Suppl. Ageing and Social Inclusion/Exclusion 1: 137-158.

Wiles, J. L., A. Leibing, N. Guberman, J. Reeve \& R.E.S. Allen (2012). The meaning of "aging in place" to older people. The Gerontologist 3: 357-366.

\section{ENTREVUES}

Entrevues 1, 2, 4, 5 et 8 (intervenants sociaux de Robertville)

Entrevues 10, 11, 12, 14, 15, 16 et 17 (intervenants sociaux de Saint-Wilfred) 